\title{
Analytics for Autonomous C4ISR within e-Government: a Research Agenda
}

\author{
Trevor J. Bihl \\ Air Force Research Laboratory, USA \\ Trevor.Bihl.2@us.af.mil
}

\author{
Michael Talbert \\ Air Force Research Laboratory \\ Michael.Talbert@us.af.mil
}

\begin{abstract}
e-Government enables big data analytics to support decision processes in governing. C4ISR (Command, Control, Communications, Computers, Intelligence, Surveillance and Reconnaissance) is essentially $e$ Government scoped to military decision processes. The value of big data and its challenges are common to both. High variety and demand for veracity compel domain expertise-specific data analysis, and increasing volume and velocity hinder data analytics at scale. These conditions challenge even highly automated methods for comprehensive cross-domain analytics, and motivate cognitive approaches such as underlie Autonomous Systems (AS) aimed at C4ISR. A C4ISR framework is examined by parts, linking each $C$ to ISR capability, and a taxonomy of analytics is extended to include cognitive autonomy enablers. Coupling these frameworks, the authors propose an extension of cognitive approaches for autonomy in C4ISR to e-Government in general and outline a research agenda for attaining it.
\end{abstract}

\section{Introduction}

e-Government supports the complex, overlapping, and conflicting processes of governing. Data analytics support e-Government. As governing increases in scope and complexity in response to demographic, economic, environmental, and political dynamics, so the value of data increases. "e-Governing” then draws on increasing variety, veracity, volume, and velocity of data -- big data. Here the term refers to the condition where the " 4 Vs" challenge the ability to analyze it in operationally required timelines [4]. Within e-Government processes, traditionally military-oriented C4ISR approaches can guide data collection, evaluation and interpretation to provide decision-supporting responses relevant to operational timelines. We examine data analysis methods in government services to see how.

e-Government services (GOV) have been described as existing within six general " $G 2 x$ " categories [5] [6]:

- GOV Delivering Services to Individuals (G2IS)

- GOV to Individuals for Political Processes (G2IP)

- GOV to Business as a Citizen (G2BC)

- GOV to Business in the Marketplace (G2BMKT)

- $\mathrm{GOV}$ to Employees (G2E)

- GOV to Government (G2G).

Such service categories relate ultimately to valuegeneration from data collectors to data consumers. As might be expected, in all of these functions, data is collected in increasing quantities and from increasingly diverse sources. Individually and collectively, these services are subject to degrees of big data challenges.

Among military-related e-Government processes, C4ISR (Command, Control, Communications, Computers, Intelligence, Surveillance, and Reconnaissance) implies both an architecture and a set of capabilities for analyzing data to provide situational awareness and decision aiding [7]. In a C4ISR framework, big data analytics derive meaning in data and help answer (and reveal) questions. As a militaryspecific set of G2G services, C4ISR relies on infrastructure to collect, communicate, analyze, and act accountably based on data. So it is with the other " $\mathrm{G} 2 \mathrm{x}$ " services. Indeed two fundamental similarities with eGovernment services and C4ISR processes is the supreme value of data and the challenges of handling it - collection, curation, correlation, and computation to derive value. We propose then, that e-Government in general can benefit from emerging advances in data processing approaches in C4ISR.

Data, information, knowledge and wisdom are generally related as depicted in Figure 1, where, e.g., wisdom is considered knowledge plus insight [8].

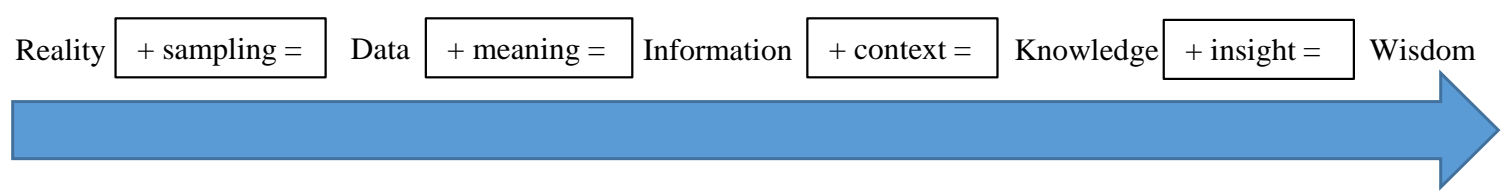

Figure 1. General relationship between reality and the data, information, knowledge and wisdom (DIKW) hierarchy, adapted from [1] [2] [3]. 
However, finding insight and meaning from data, i.e. extracting knowledge and wisdom, requires human expertise in the analysis loop. Analytics can process data, facilitate human interpretation, and aid knowledge and wisdom development. However, as big data challenges increasingly affect e-Governing disciplines, humans will only with greater difficulty and perhaps less efficiency, exhaustively evaluate relevant data. When human ability is saturated and automation lacks wisdom, autonomous analysis methods could well become a practical necessity.

Machine autonomy goes beyond automation, the latter acting in well-defined operating conditions (OCs) and more open to human-in-the-loop decisions [9]. Autonomous systems (AS) have three distinctive characteristics: 1) intelligent, informed, and unforced choices, 2) an ability to handle uncertain and unexpected situations, and 3) a sense of self [9]. In a C4ISR context, AS could be incorporated in both the ISR approaches, i.e. data analytics, as well as $\mathrm{C} 4$ components, i.e. for onboard or offboard decision making and controls. The authors envision an effective autonomous approach to C4ISR where human operators are at an executive level of Command and Control (C2). Presently, C4ISR operations involve a human analyst considering data from various sources, e.g. G2G, and imparting agency to virtual or physical assets via C2. Noting that C4ISR occurs on a range of time-scales, from real time, total system resource management, to longer latency forensic analysis, in the rest of this work, we explore possibilities of autonomy-enabling analytics to autonomous decision making across the spectrum of decision timelines. By expanding the C4ISR components to include autonomy enabling analytics and processes, we motivate that processes can be similar at both ends of the time scale.

\section{C4ISR and e-Government}

C4ISR, as an architecture, at its core encapsulates two components: resources and capabilities. Resources are the $\mathrm{C}(*)$ components, e.g. command, control, communications, and computations; capabilities from expending $\mathrm{C}\left({ }^{*}\right)$ resources are the information-based decision aiding processes and processes.

C4ISR results in synthesized products where information is gained through ISR (collection and analytics) activities. ISR activities can range from CPUbased analysis of G2G data products to real-time exploitation of streaming sensor feeds [7]. In conducting ISR, data science-based analytics can be used by operators and automated onboard systems to provide awareness- and decision-aiding data for inference [7].

In C4ISR, the authors adopt the hierarchical process of data engagement in [10] and adapt it as in Figure 2. In [10], a general e-Government hierarchy of data, agents and services is presented. It links the data sources to services, which access and process the data, as well as agents who analyze, collect, and consume the data.

Figure 2 illustrates the first proposed extensions from [10] -- overlaying the DIKW hierarchy from Figure 1. In Figure 2, data source consumers query sources of data via services. Data products and services are refined through man-machine operations providing analytics to aid analysts. This enables e-Government.

\subsection{G2G e-Government}

In adopting a C4ISR approach for G2G systems, one must address crucial V's of big data: volume, variety, and velocity [4]. Regarding the variety of data sources, one must work to ensure availability and accessibility across silos, even single source ones. As discussed in [10], linked data and adherence to standards can address this. Further, as in Figure 2, while data variety is a notable aspect of the data in the general e-Gov catalog, considerations must also be made for the variety of agents accessing data: human analysts, machine analytics, providers, consumers, and "prosumers". Data availability and interoperability concerns also exist in data collected and shared in G2G domains [11][12], however, G2G projects focused on data ingestion have addressed this via architectural approaches [12]. Beyond data variety, increasing volume and velocity (i.e. scale) of big data in G2G services increase the processing and analysis challenges to human analysts. Intelligent analytics and autonomy become essential to timely operational solutions, and underscore the $4^{\text {th } ~ ' ~} \mathrm{C}$ ' in the C4ISR paradigm ('computing').

\subsection{C(*)ISR as a functional paradigm}

In this section, we examine by parts the $\mathrm{C}(*)$ resources to ISR capabilities introduced earlier. First, we assert as a construct and for clarity, ISR capabilities imply coarsely, a scale and scope of information gained:

- $\quad \mathrm{R}$ - Reconnaissance - examination and analysis examination of a location at discrete points in time

- S - Surveillance - continuous examination and analysis of an area over time

- I - Intelligence - comprehensive, coherent, and predictable pattern synthesis from $\mathrm{S}$ and $\mathrm{R}$.

In Table 1 we notionally express ISR-enabling contributions of the $\mathrm{C}(*)$ capabilities.

Table 1. Linking C(*) to ISR capabilities

\begin{tabular}{|l|c|c|c|}
\hline & Reconnaissance & Surveillance & Intelligence \\
\hline Command & location & duration & motivation \\
\hline Control & frequency & resource mgt & scope \\
\hline Comm. & data batches & data streams & disseminate \\
\hline Computers & state analysis & scene analysis & prediction \\
\hline
\end{tabular}




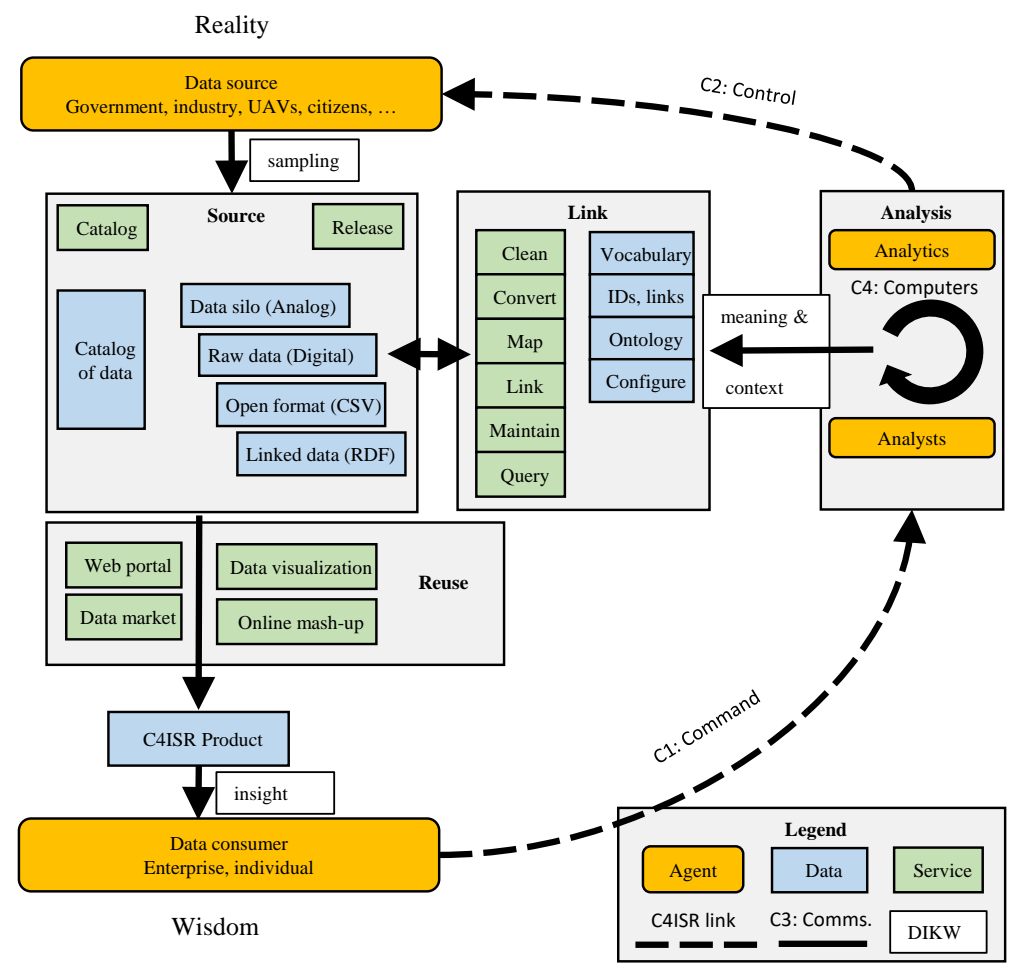

Figure 2. Roadmap of e-Government Data, adapted and expanded from [10] to include both a mapping to C4ISR and DIKW components. C3 (communications) permeates the roadmap.

The authors here posit that the C's are the raw abilities that yield useful ISR capabilities. That is, allocating resources (manpower, funds, time, etc.) to the C's can yield increasing capabilities in ISR. Stated another way, one can view the general relationship as

$$
\text { Quality }=\frac{\partial(I S R)}{\partial C(*)}
$$

encapsulating the plain understanding of Table 1 that the capabilities yielded from ISR are realized through investments in $\mathrm{C}(*)$. Quality, then, varies with degree.

Notably, many $\mathrm{C}(*)$ components are manpower intensive, particular the C2 component, and this resource is available at a premium. Thus, the authors envision that advanced computing capabilities are needed to offset manpower constraints. Thus, the understanding in (1) can be further expended to encompass expenditures in technology (Tech):

$$
\text { Gain }=\frac{\partial}{\text { Tech }}\left(\frac{\partial(I S R)}{\partial C(*)}\right)
$$

providing an illustration of how technology can influence the gain in capabilities.

The authors further envision that advanced computing capabilities, enabling advanced analytics, can facilitate manpower moving from a C2 role to a primarily C1 (command) role. In the following sections, this vision and its components will be explored.

\section{Data Science and Analytics}

Analytics are considered herein as the techniques used to explain, model, and analyze data. Considered broadly, the authors consider those associated with data science, statistics, pattern recognition, machine learning, and related domains, c.f. [13] [14] [15].

As seen in Figure 2, analytics is necessary to provide solutions and services from data. C4ISR processes incorporate many analytic abilities, but require human analysts for synthesis leading to decisions and resource commitment. Current analytics methods examine data through the use of rules and logic, which are either developed through algorithmic means (Machine Learning) or designed into the system (Expert Systems). While these methods can be very sophisticated, all such algorithms fail when presented with data outside the bounds of its training, e.g. [17] [18]. However, human cognition (as wisdom) routinely excels and adapts to such situations. E.g., humans would rarely confuse a gibbon with a panda yet machine learning algorithms frequently do [19] [20]. 


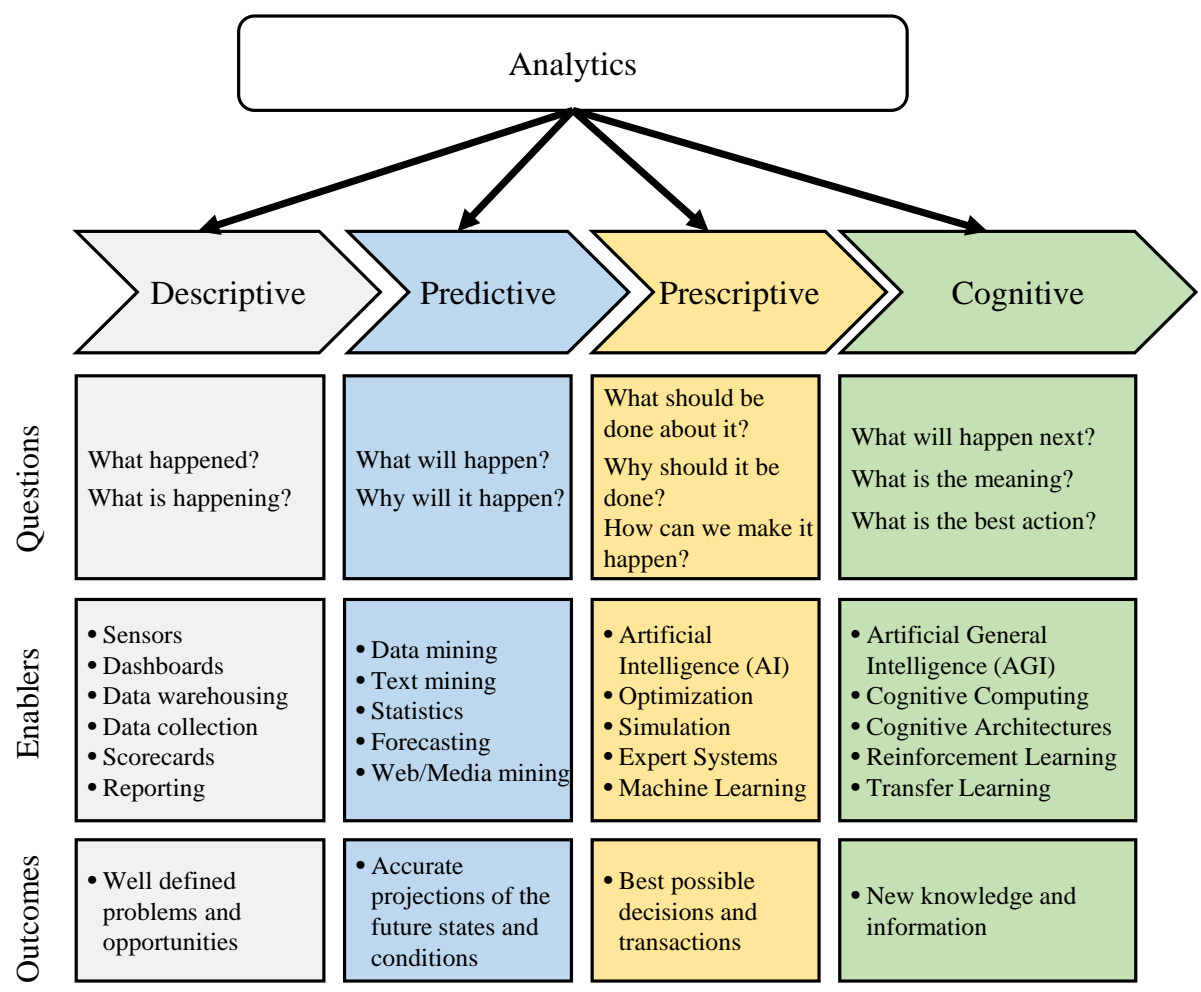

Figure 3. Taxonomy of Analytics for Autonomy, adapted and expanded from [16].

Additionally, big data in enterprise scale organizations causes "enterprise amnesia" wherein the data collected outstrips algorithmic abilities [21]. When, as is occurring now, big data holdings in G2G eGovernment grow on par with the expansion of data holdings in other national sectors, intrinsic, organic, self-servicing and even self-healing solutions are warranted. These are features of organisms, and not short of what we ultimately need in autonomous solutions for e-Gov big data challenges.

While advanced analytics, often called artificial intelligence (AI), are highly sophisticated, they are the result of complex automated algorithms. Such methods become brittle when unexpected information is processed, e.g. [18], and even powerful analytics methods need human input for synthesis and understanding, especially for timely, responsible, and even grave decisions. In the last remark we imply degrees of autonomy. We explore this below.

\subsection{Taxonomy of Analytics for Autonomy}

Typical taxonomies of analytics, e.g. [16], include descriptive, predictive, and prescriptive levels, as captured in the first three columns of Figure 3. Descriptive analytics consider answering questions about what happened or is happening and encompass sensor data logging and methods to describe the general data structure, e.g. measuring central tendency and dispersion of data [16]. Predictive analytics extend upon descriptive analytics and answer questions such as "what will happen?" and involve more advanced algorithmic methods to model the structure of the data and provide inference abilities [16]. Prescriptive analytics further expand upon the analytics base and start to answer questions such as "what should be done?" and these involve more sophisticated methods to model the data and prescribe the correct decisions [16].

However, for autonomous systems, further capabilities are needed. Missing in the taxonomy of [16] is the ability to handle unexpected events/data and create new knowledge/information. Accounting for this, we add cognitive analytics as an extension of the analytical foundation [15]. Cognitive analytics generally include capabilities such as knowledge representation, inference, reasoning, learning, and hypothesis generation [15]. At a high level of capability, cognitive analytics embody artificial general intelligence (AGI), which can be considered as building AI with reasoning, cognition, and rationality [22]. Performed correctly, cognitive analytics have the potential of enabling machine computation to reach the knowledge level of the hierarchy presented in Figure 1. Using this understanding, Figure 3 depicts an extension of taxonomy of [16] to include cognitive analytics effected by autonomous processes. 


\subsection{Autonomy}

Autonomous capabilities are needed for advanced analytics wherein an analyst could consider the inferential results from multiple analytical agents. To understand what is needed for autonomous analytical agents, one must understand the current state of the art for machine intelligence with respect to automation and autonomy. One can consider automation, as the following definition, from [20]:

1. Automation: The system functions with no or little human involvement, however performance is limited by the specific logic it has been designed to employ, and the actions it has been designed to do. Typically these are well-defined tasks that have predetermined outcomes, i.e. rule-based responses.

Notably, this does not include the ability to anticipate or address uncertainty, an ability of living organisms and autonomous systems. In this perspective, a working definition for autonomy can be considered, per [20], as:

2. Autonomy: Systems which have intelligencebased capabilities, allowing it to respond to situations that were not preprogrammed or anticipated in the design. Autonomous systems have a degree of self-government, self-directed behavior (being the human's proxy for decisions).

Thus, inherent in an autonomous system are three general characteristics: 1) intelligence, 2) an ability to handle unexpected situations, and 3) a notion of self [9]. Inherently, these characteristics are those of cognition. To provide autonomous operations a system needs "cognitive autonomy," which involves the ability to resolve conflicting information, plan, and learn [23].

Stated another way, autonomous analytics can only be achieved when goals, tasks, cognition, and command abilities are enabled such that an AS can select the appropriate task or goal to pursue, modify its thinking constructs, and appropriately assume roles [9]. However, achieving operational autonomous analytics is difficult and fundamental research must be conducted.

\section{A Research Agenda for C5ISR}

The authors envision and propose autonomous analytics as providing a realization of C5ISR where the $\mathrm{C}(5)$ is cognition, the primary necessary component of autonomy. However, to fully realize C5ISR involves creating more than cognitive analytics. It includes extensions of C4ISR to account for human-machine teaming (HMT) and human-machine interfaces (HMI). Still further, it requires metrics to evaluate autonomy component and HMT effectiveness, as well as to provide assurances for bounds on autonomous system behaviors. In addition to leveraging the foundational works identified so far, in developing a research agenda for C5ISR the authors leverage recent U.S. Government frameworks including the USAF Autonomous Horizons Vol. 2 [24], the challenge areas identified in the DOD Autonomy Community of Interest [25], discussions on autonomous vehicle architectures [26], and principles embodied in a research agenda on HMT [27].

In order to develop and implement C5ISR, the authors outline a research agenda in Figure 4. There are three components: Reasoning, Implementation, and Application. Now we look more closely at each component.

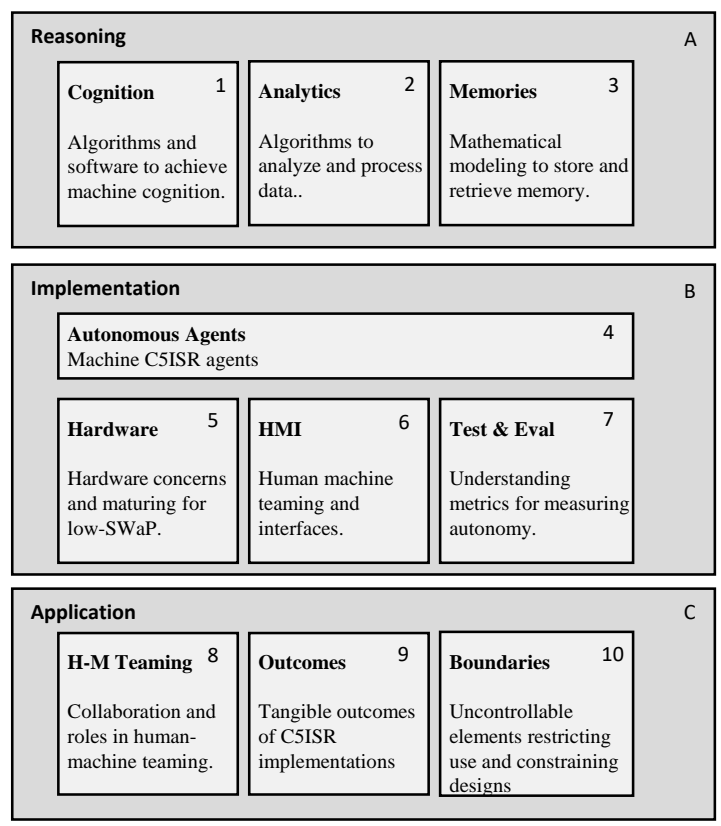

Figure 4. Research Agenda for C5ISR

\subsection{Reasoning (Meta Theme A)}

Within Figure 4, Reasoning encompasses research and development (R\&D) in machine perception, reasoning, and intelligence. This includes cognitive analytics and analytics in general, cognitive architectures, memories and representation, and situation understanding.

Reasoning addresses 1) extending the analytics taxonomy of Figure 3 to include autonomy enabling capabilities, this further includes 2) architectures which mimic and replicate biological brains for decision making, and 3) memory structures to recall and store data. In order for a machine to reason, this implies the ability to learn, adapt, and handle unexpected events, e.g. much beyond what an algorithm was trained on and beyond the current state of the art in machine learning. 
It is known that biological brains employ high efficiency and compress sensed data at a very high rate. The human mind is able to collect up to $10^{7}$ bits of information yet process at 25-75 bits/sec (rate depending on channel -- auditory, visual, tactile, etc.) [28] [29]. This rate eclipses even big data analytics high volume burst capabilities, which still are not scalable for always-on AS. Clearly, speed and scale approaching biological system abilities for efficient storage, retrieval, and processing is required.

4.1.1. Cognition (Theme 1). Machine cognition, cognitive analytics, and cognitive architectures aim to leverage this foundational work in neurosciences to develop approaches for effective and efficient AI. There have been some successes in this area, including the development of the LIDA architecture and its deployment on the US Navy's G2E personnel system [30]. However, LIDA's G2E application was aimed at a specific problem while a C5ISR approach would need to apply to many different problems and events, even with a potential to expand in scope, within the same general architecture.

Additionally, current research in machine autonomy involves components such as cognitive architectures, which provide an infrastructure for AS and learning systems [31]. Still, though practical cognitive architectures, e.g. [30] [32], have seen use in decision making, reasoning, and inference, issues exist in scalability, timeliness, and efficiency when these are employed on a large scale [32].

A variety of research questions remain in this area:

- What are the appropriate reasoning and logical constructs for a cognitively flexible AS?

- How can cognitive architectures be combined with natural language processing for bidirectional communication and learning?

4.1.2. Analytics (Theme 2). In addition to cognitive analytics as a general topic, primary concerns for analytics in general for C5ISR include developing abilities to handle unexpected events. Notably, some work has been done to date in this area, including: analogical reasoning [33], out-of-library considerations [34], and transfer learning [35]. However, capabilities are still limited as noted in [24]. A variety of research questions remain in this area:

- How can analytics methods be extended to handle conditions outside the training space?

- What is needed to enable, record, and reuse experiential learning in AS for analytics?

- How can an AS respond to changes in mission objectives by task-flexible selection of analytics?
4.1.3. Memories (Theme 3). Currently available AI systems generally aim to collect and store all possible data in as high dimensionality as possible. This becomes highly inefficient as a system scales. We know that biological cognition is very efficient in this area, and thus a particular focus is needed on new approaches for machine memory storage and retrieval. This work currently focuses on procedural, declarative, and working memories and their effective interactions. A variety of research questions remain in this area:

- How can memories (and knowledge gained) from past events be stored efficiently?

- What approach to management of memories (e.g. procedural, declarative, and working) and their interactions ensures timely and viable AS?

\subsection{Implementation (Meta Theme B)}

Implementation involves $R \& D$ in hardware tradeoffs, HMIs for appropriate user operations, and test and evaluation for developing and understanding metrics for assessing C5ISR performance.

4.2.1. Autonomous Agents (Theme 4). Beyond developing components for an AS agent, integrating components and developing the agent itself is needed for effective C5ISR use. Questions for this area:

- What computational and reasoning components comprise effective AS agents and HMT?

- How to define a component trade-space to predict system performance relative to mission objectives?

- What is the effect of levels of peer interaction on operational trust-ability of the AS agent?

4.2.2. Hardware (Theme 5). Beyond developing an AS for C5ISR, implementing it with users and infrastructure in mind is necessary. In this, hardware constraints, i.e. Size, Weight, and Power (SWaP), must be considered when discussing computing since cognition is computationally expensive [36]. With high-SWaP capacity, e.g. dedicated on-demand supercomputer time, little constrains system. In contrast, consider niche systems, e.g. autonomous vehicles or single CPU computers, where SWaP constraints may limit utility and capabilities, since power budgets may not support raw demands of algorithms or high volume data throughput, or on-board cooling, or any two or all three.

This is key for any proposed C5ISR implementation since cognition is computationally expensive, e.g. [36], and computation comes at a cost. General e-Gov systems will likely operate with unconstrained SWaP provisions (i.e., in a permanent building with continuous power). However, given the diversity of systems and subsystems and their ages and SWaP requirements, 
SWaP-C considerations should apply across the e-Gov when evaluating what systems should be upgraded (or downgraded), improved, or replaced. Besides desired "greening," sustainability and possibly security may be enhanced by the right SWaP.

Some solutions to SWaP concerns relate to selecting the appropriate method for the task at hand, as in [36]. Another solution involves incorporating "AI Accelerators.” [15], advanced electronics to get around a Moore's Law bottleneck on computation abilities available on CPUs. As presented in Table 2, AI accelerators include technologies and capabilities such as current parallel computing and graphics processing units (GPU), in- development neuromorphic chips, and potentially realizable quantum computing. Considering the foregoing and hardware maturity and SWaP concerns have enumerated in other technology reviews, e.g. [37]. Hardware-related research questions include:

- What AI accelerators best enable a given missiondriven reasoning and HMT implementation?

- Which of size, weight, and power are likely to produce highest gains in AS enablement if reduced, made more efficient, or less costly?

Table 2. Example AI accelerator technologies with potentials to facilitate future C5ISR abilities

\begin{tabular}{|l|c|c|}
\hline & Maturity \& SWaP & Ref. \\
\hline Parallel computing & High maturity, High SWaP & {$[38]$} \\
\hline GPU & High maturity, High SWaP & {$[39]$} \\
\hline Neuromorphic chips & Med maturity, low SWaP & {$[40]$} \\
\hline Memristors & Low maturity, low SWaP & {$[41]$} \\
\hline Quantum computing & $\begin{array}{c}\text { Very low maturity, } \\
\text { theoretically low SWaP }\end{array}$ & {$[42]$} \\
\hline
\end{tabular}

4.2.3. Human-machine interaction (HMI) (Theme 6). Human-machine interaction (HMI) in AS is of perhaps greater interest than the machine-machine interactions in hardware alone, owing to the external fragility and internal complexity of the human. HMI involves the complex yet cooperative interaction between humans and system components necessary to facilitate collaboration and coordination [43] [44]. HMI includes both technical and non-technical accommodations and often employs a user-centric approach [43] [24]. Of primary interest is reducing user workload and displaying information helpfully [45]. The latter is challenged by increasing data volume, velocity, variety which additionally complicates the analytics and reduces the time available to process the data. We consider these research questions:

- How to effectively relay AS internal situation assessment, i.e., beliefs and thoughts, to users for effective peer flexible interaction?

- Can an HMI enable trust between users and autonomous systems for effective teaming?
- How to develop training for the user and the AS for joint, mutual, and individual benefit?

4.2.4. Test \& Evaluation (Theme 7). Finally, if the hardware works and the human has a functional partner in the AS machinery, how well does the total system accomplish the mission? Test and Evaluation includes understanding how to evaluate an AS and develop trust. Current issues in AI, AGI, and AS include unexplainable results and explainable AI (XAI) has recently been a large research goal [24]. While XAI approaches can extend into fully explainable results, largely what is of interest is trustable and reliable AS. This can be thought of in much the same way as service dogs which, though trained and trusted, are still black boxes since they cannot be queried or questioned.

Beyond this, understanding when and where an AS should be used is important. In some applications, it is logical that a human is more efficient at the task; but, the division between such tasks is not yet obvious beyond coarse divisions. For us, research questions include:

- What measures quantify AS-human teaming effectiveness and their respective contributions?

- How to quantify human-agent interaction quality in an AS, perhaps even a function of mission?

- What measures quantify an AS's trusted ability to act as a human proxy for timely, critical missions?

\subsection{Application (Meta Theme C)}

Application involves understanding how to decentralize tasks effectively, how to enable effective HMT, and what outcomes and products are possible. Extending the implementation, application involves deploying and employing a trusted AS with appropriate human-agent teaming.

4.3.1. Human Machine Teaming (Theme 8). Beyond developing an AS for C5ISR, implementing it within an HMT team is critical. Here, an effective HMI is used, but now conditions outside the training space are possible since the system is used outside the developmental context. Upon employment, effective analysis of outcomes and relaying results back to developmental efforts drives improvement. To that end, these questions apply:

- How to measure the quality of live experiential learning, outside the test environment?

- Can a trustable AS be matured with the experience of live experiential learning?

- How does the mission drive the division of labor?

4.3.2. Outcomes (Theme 9). In evaluating the performance of an AS and HMT C5ISR, one must 
consider the mission outcomes as both tangible and intangible work products [27]. Research questions about outcomes include, for instance:

- How to measure intangible outcomes e.g., perceived goal attainment and user satisfaction?

- What further intangible outcomes are measureable (e.g., user experience)?

- What is the overall utility of the AS with respect to mission timeliness, task diversity, and criticality?

4.3.3. Boundaries (Theme 10). Safety, trust, legal, and usability boundaries all exist within the scope of deploying an AS in an HMT for C5ISR. Currently, no safety standards exist for ASs, and currently available safety standards, e.g. IEC 61508 and ISO 13849-1, assume direct human involvement, which cannot be assured when an AS acts as a human proxy [26]. Additionally, the legal questions regarding the liability of decisions made by an AS are unsettled [46]. Thus, leveraging [27], various research questions include:

- What safety standards, policies, and legal restrictions need development for AS use?

- What cultural norms, values, and behavioral expectations constrain developing AS agents?

- What as-yet unnoticed boundary may block or advance the use of AS agents and HMT?

\subsection{Developing Performance Metrics for C5ISR}

In developing technologies and capabilities for C5ISR, (recalling the fifth ' $C$ ' is 'cognitive'), that extend to general e-Governing, specific and appropriate measures are necessary to evaluate results. Used effectively, the analysis of assessed performance can also guide technology development and inform research agendas [9] [47]. We propose to let the relationships in eqs (1) and (2) serve as a guide to assessment:

- Has desired product or service quality been delivered?

- Has an achieved gain been sufficient to the need?

While the application of metrics is well studied in many deterministic applications, e.g. text and data analytics performance measures [48], areas such as autonomy are not. The authors present two classes of metrics to round out the research agenda.

4.4.1. Autonomy Evaluation Metrics. Various approaches exist to quantify how autonomous an agent is, e.g., the SAE J3016 approach for quantifying the autonomy of cars. However, limitations exist in many approaches due to their discrete nature or limited granularity [9]. Of particular interest in [9] is creating an initial approach to synthesizing the literature on autonomous system metrics, resulting in a suggestion to use metrics that have concise definitions, are progressive in nature (ideally continuous), are verifiable, use well-defined and measurable inputs to facilitate implementation, and are simple to understand.

Additionally, in [9] the authors identified three flexibilities that characterize autonomous behavior. These three flexibilities can be defined, using the short and concise definitions of [49], as

- Task flexibility: the ability of an agent to identify, select, act, and complete multiple tasks to complete a mission or activity

- Peer flexibility: the ability of an agent to change from subordinate, peer, and supervisory roles as necessary to complete a mission or activity

- Cognitive flexibility: the ability of an agent to change decision boundaries, rules, or models for a given task, e.g. adaptive cognition.

The source cited recommends measuring capabilities of an agent (model building, knowledge forming, knowledge transferring, acting, simulating, planning, and understanding) as they are applied to the three flexibilities [9].

4.4.2. Deadline, Responsibility, and Gravity (DRaG). Beyond developing capabilities to enable C5ISR, we need guidelines for when and where to best use autonomous solutions versus human-in-the-loop. This is largely a matter of trust in an AS as well as appropriate use of teammates (humans, AS, and human-agent teams) given demonstrated abilities vs. required tasks.

To evaluate these considerations, the authors leverage stakeholder theory, which provides the following attributes of stakeholders to a situation: power (to influence), legitimacy (of the relationship), and urgency (of the claim) [50]. Modulating these ideas, the authors have identified three areas that are of a concern when using AS and human-AS teams. These are:

- Deadline of the decision to be made. For real time, continuous, dynamic actions an AS may well be better suited than asking a user and waiting for a response, repeatedly.

- Responsibility, i.e. who has ownership of the decision, and perhaps even the legal standing. E.g., an AS might not be permitted to declare guilt (involving humans), but it could logically identify potentially criminal activity from forensic analysis of financial transactions, or enemy military vehicles in a battleground from imagery or signals.

- Gravity, a general understanding of risks and tradeoffs. For example, a trusted low gravity decision might be best tendered by an autonomous system since 1) the risk of a bad outcome is minimal, and 2) it reduces the human cognitive 
burden, especially if the decision is called for with high frequency, is excessively broad in scope (even if bounded), or is mind-numbingly repetitive.

Table 3. Example DRaG analysis possibilities and implications

\begin{tabular}{|c|c|c|c|c|}
\hline Deadline & Respons. & Grav & $\begin{array}{c}\text { Motivating } \\
\text { Example }\end{array}$ & $\begin{array}{l}\text { C5ISR Potential } \\
\text { for AS (outcome) }\end{array}$ \\
\hline Fast & Machine & Low & $\begin{array}{c}\text { GIS Mapping } \\
\text { services }\end{array}$ & $\begin{array}{c}\text { acts and logs } \\
\text { (annotated maps) }\end{array}$ \\
\hline Fast & Human & Low & $\begin{array}{c}\text { G2IS notary } \\
\text { services }\end{array}$ & $\begin{array}{l}\text { acts and notifies } \\
\text { (notarized docs) }\end{array}$ \\
\hline Slow & Machine & Low & $\begin{array}{c}\text { FHA/VA loan } \\
\text { approvals }\end{array}$ & $\begin{array}{c}\text { acts and logs } \\
\text { (load decisions) }\end{array}$ \\
\hline Slow & Human & Low & Issue Passports & $\begin{array}{c}\text { acts and logs } \\
\text { (passport) }\end{array}$ \\
\hline Fast & Machine & High & $\begin{array}{c}\text { FAA Collision } \\
\text { avoidance }\end{array}$ & $\begin{array}{l}\text { acts and notifies } \\
\text { (disaster averted) }\end{array}$ \\
\hline Fast & Human & High & First Responders & $\begin{array}{c}\text { presents options } \\
\text { (triage) }\end{array}$ \\
\hline Slow & Machine & High & $\begin{array}{c}\text { Congressional } \\
\text { Budgeting } \\
\end{array}$ & $\begin{array}{l}\text { analyzes options } \\
\text { (feasible budget) }\end{array}$ \\
\hline Slow & Human & High & $\begin{array}{c}\text { Military } \\
\text { operations }\end{array}$ & $\begin{array}{l}\text { predicts outcomes } \\
\text { (resource phasing) }\end{array}$ \\
\hline
\end{tabular}

When considering $\mathrm{DRaG}$, one thus has three axes to assess and attempt to optimize. A conceptualization of a DRaG analysis with possible implications and examples is presented in Table 3 . Table 3 decisions and services for pairwise combinations of DRaG factors ordered by "gravity". Within Table 3, motivating examples are listed of current e-Gov processes, performed by either humans or automation. In Table 3, the C5ISR potential is given for the motivating examples which relates to how a C5ISR AS would act given the DRaG. In parentheses are the potential outcomes of C5ISR action.

\section{Conclusions}

The authors motivate here how the C4ISR eGovernment (primarily military) architecture can be extended to address challenges inherent in big data analytics. Further, the authors illustrate how a C4ISRlike architecture is linked inherently to general eGovernment systems, and that some e-Government functions stand to benefit from enabling autonomous systems (AS) to assist in big data related processes.

To tackle this problem, the authors propose a research agenda for C5ISR where $\mathrm{C}(5)$ is cognition. Through an understanding of (Gov-to-Gov) G2G concerns, the authors depict a hierarchy of analytics to include cognitive analytics for improved G2G data analysis to process big data in a C4ISR structured enterprise. By incorporating cognitive analytics, the authors propose that C5ISR can abide and provide autonomous processing of data and the autonomous delivery of data products. Finally, the authors further present C4ISR as a functional paradigm whereby different C's enhance ISR capabilities. We note challenges in employing C5ISR solutions and present an array of evaluation approaches when developing AS. Key research questions are provided.

\section{References}

[1] M. Jennex, "Re-visiting the knowledge pyramid," Hawaii Int. Conf. on System Sciences, pp. 1-7, 2009.

[2] M. Frické, "The knowledge pyramid: a critique of the DIKW hierarchy," J. of information science, vol. 35, no. 2, pp. 131-142, 2009.

[3] S. Carpenter, "A Primer: Enterprise Wisdom Management and the Flow of Understanding.," CognitiveCybernetics.com, 2008.

[4] T. Bihl, et al., "Defining, Understanding, and Addressing Big Data," Int. J. of Business Analytics (IJBAN), 3(2), pp. 132, 2016.

[5] L. Carter and F. Bélanger, "The utilization of e-government services: citizen trust, innovation and acceptance factors," Information Systems J., 15(1), pp. 5-25, 2005.

[6] F. Belanger and J. Hiller, "A framework for e-government: privacy implications," Business process management J., vol. 12, no. 1, pp. 48-60, 2006.

[7] M. Talbert and G. Seetharaman, "When sensor Webs start being taken seriously," IEEE Int. Conf. Sensor Networks, Ubiquitous, and Trustworthy Comput. (SUTC'06), 2006.

[8] D. Stenmark, "Information vs. knowledge: The role of intranets in knowledge management," Hawaii Int. Conf. on System Sciences, pp. 928-937, 2002.

[9] T. Bihl, et al., "Finding common ground by unifying autonomy indices to understand needed capabilities," SPIE Proc., 2018.

[10] L. Ding, et al., "Linked open government data," IEEE Intelligent systems, 27(3), pp. 11-15, 2012.

[11] N. Wisitpongphan and T. Khampachua, "Impact of Globalization on Interoperability in Digital Government," Proc. of 17th European Conference on Digital Government ECDG, pp. 254-261, 2017.

[12] D. Maluf and D. Bell, "Towards G2G: systems of technology database systems," IEEE Aerospace Conference, pp. 4320-4326, 2005.

[13] M. Waller and S. Fawcett, "Data science, predictive analytics, and big data: a revolution that will transform supply chain design and management," J. Business Logistics, 34(2), pp. 77-84, 2013.

[14] A. Gandomi and M. Haider, "Beyond the hype: Big data concepts, methods, and analytics," Int. J. Inform. Management, 35(2), pp. 137-144, 2015. 
[15] V. Gudivada, M. Irfan, E. Fathi and D. Rao, "Cognitive analytics: Going beyond big data analytics and machine learning," Handbook of Statistics, vol. 35, pp. 169-205, 2016.

[16] D. Delen and H. Demirkan, "Data, information and analytics as services," Decision Support Systems, vol. 55, pp. 359-363, 2013.

[17] A. Krizhevsky, et al., "Imagenet classification with deep convolutional neural networks," Adv. in Neural Inform. Processing Systems, pp. 1106-1114, 2012.

[18] C. Szegedy, et al., "Intriguing properties of neural networks," arXiv preprint, 2013.

[19] I. J. Goodfellow, J. Shlens and C. Szegedy, "Explaining and harnessing adversarial examples," arXiv preprint arXiv:1412.6572, 2014.

[20] E. Blasch, et al. Fenstermacher and R. Patterson, "QuEST for information fusion," IEEE National Aerospace and Electronics Conf., pp. 215-223, 2014.

[21] J. Jonas, "Enterprise Amnesia vs. Enterprise Intelligence.," Emetrics, 2011.

[22] P. Wang, "What Do You Mean by “AI”?," Proc. of the First AGI Conference, pp. 362-373, 2008.

[23] D. Floreano and R. Wood, "Science, technology and the future of small autonomous drones.," Nature, vol. 521(7553), p. 460, 2015.

[24] G. L. Zacharias, Autonomous Horizons The Way Forward, Maxwell AFB: Air University Press, 2019.

[25] J. Bornstein, DoD Autonomy Roadmap: Autonomy Community of Interest, Aberdeen, MD: Army Research Laboratory Aberdeen Proving Ground, 2015.

[26] S. Behere, et al., "Architecture challenges for intelligent autonomous machines," Intelligent Autonomous Systems, pp. 1669-1681, 2016.

[27] I. Seeber, et al., "Machines as teammates: a collaboration research agenda.," Hawaii Int. Conf. on System Sciences, pp. 420-429, 2018.

[28] B. Mortimer and L. Elliott, "Context Sensitive Tactile Displays for Bidirectional HRI Communications," Adv. Human Factors in Robots and Unmanned Sys., pp. 17-26, 2017.

[29] D. Donoho, "Sparse components of images and optimal atomic decompositions," Constructive Approximation, 17(3), pp. 353-382, 2001.

[30] S. Franklin and F. Patterson Jr, "The LIDA architecture: Adding new modes of learning to an intelligent, autonomous, software agent," Integrated Design and Process Technology, pp. 1-8, 2006.

[31] P. Langley, et al., "Cognitive architectures: Research issues and challenges," Cognitive Systems Research, 10(2), pp. 141-160, 2009.

[32] C. Eliasmith, How to build a brain: A neural architecture for biological cognition., Oxford University Press., 2013.
[33] C. Eliasmith and P. Thagard, "Integrating structure and meaning: A distributed model of analogical mapping," Cognitive Science, 25(2), pp. 245-286, 2001.

[34] T. Albrecht and K. Bauer, "Optimization framework for a multiple classifier system with non-registered targets," $J$. Defense Modeling and Sim., 5(1), 2008.

[35] S. Pan and Q. Yang, "A survey on transfer learning," IEEE Trans. on knowledge and data eng., vol. 22, no. 10, pp. 1345-1359, 2009.

[36] K. Thórisson and H. Helgasson, "Cognitive architectures and autonomy: A comparative review," J. Artificial General Intel., vol. 3, no. 2, pp. 1-30, 2012.

[37] P. Blouw, et al., "Benchmarking Keyword Spotting Efficiency on Neuromorphic Hardware.," arXiv preprint arXiv:1812.01739., 2018.

[38] K. Asanovic, et al., "A view of the parallel computing landscape," Commun. of the ACM, 52(10), pp. 56-67, 2009.

[39] J. Owens, et al., "GPU computing," Proc. IEEE, 96(5), 2008.

[40] C. Lin, et al., "Programming spiking neural networks on Intel’s Loihi.," Computer, 51(3), pp. 52-61, 2018.

[41] M. Prezioso, et al., ", 2015. Training and operation of an integrated neuromorphic network based on metal-oxide memristors.," Nature, vol. 521, no. 7550, 2015.

[42] T. Ladd, et al., "Quantum computers," Nature, vol. 464(7285), p. 45, 2010.

[43] H. Booher, Handbook of human systems integration, John Wiley \& Sons., 2003.

[44] M. Lohani, et al., "A framework for human-agent social systems: the role of non-technical factors in operation success," Adv. Human Factors in Robots and Unmanned Sys., pp. 137-148, 2017.

[45] A. Selkowitz, et al., "Displaying Information to Support Transparency for Autonomous Platforms," Adv. Human Factors in Robots and Unmanned Sys., pp. 161-173, 2017.

[46] A. Eugensson, et al., "Environmental, safety legal and societal implications of autonomous driving systems," Int. Tech. Conf. Enhanced Safety of Vehicles, 2013.

[47] D. Watzenig and M. Horn, "Introduction to Automated Driving," Automated Driving, pp. 3-16, 2017.

[48] T. Fawcett, "An introduction to ROC analysis," Pattern recog. let., vol. 27, no. 8, pp. 861-874, 2006.

[49] T. J. Bihl, et al., "From Lab to Internship and Back Again: Learning Autonomous Systems through Creating a Research and Development Ecosystem," Proceedings of AAAI, 2019.

[50] R. Mitchell, et al., "Toward a theory of stakeholder identification and salience: Defining the principle of who and what really counts," Academy of management review, 22(4), pp. 853-886, 1997. 